\title{
Symmetry-Breaking Bifurcation for Free Elastic Shell of Biological Cluster, Part 2.
}

\author{
Hanna Guze and Joanna Janczewska
}

\begin{abstract}
We will be concerned with a two-dimensional mathematical model for a free elastic shell of biological cluster. The cluster boundary is connected with its kernel by elastic links. The inside part is filled with compressed gas or fluid. Equilibrium forms of the shell of biological cluster may be found as solutions of a certain nonlinear functional-differential equation with several physical parameters. For each multiparameter this equation has a radially symmetric solution. Our goal is to study the bifurcation which breaks symmetry. In order to establish critical values of bifurcation parameter and buckling modes we will investigate an appropriate linear problem. Our main result on the existence of symmetrybreaking bifurcation will be proved by the use of a variational version of the Crandall-Rabinowitz theorem.
\end{abstract}

Mathematics Subject Classification (2010). 35R35, 34K18.

Keywords. Biological cluster, symmetry-breaking bifurcation, Fredholm map, free boundary problem.

\section{Introduction}

\subsection{Mathematical model and radially symmetric solutions}

In this paper we will study a bifurcation phenomenon in a two-dimensional mathematical model describing equilibrium forms of a shell of biological cluster. The cluster has an elastic boundary connected with its kernel by elastic links. The inside part of the cluster is filled with compressed gas or fluid (see Fig. 1).

An equilibrium form of the cluster is described in polar coordinates by a $2 \pi$ periodic $C^{m+2}$-smooth positive function $r(\theta), m \in \mathbb{N} \cup\{0\}$ (see Fig. 2). We derive it as a critical point of an appropriate energy functional.

The second author is supported by Grant of National Science Centre (Poland) no. 2011/03/B/ST1/04533. 


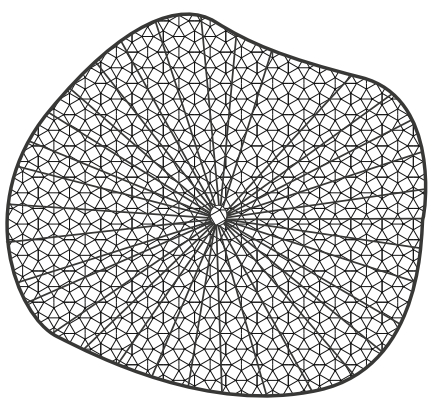

FiguRE 1. A biological cluster

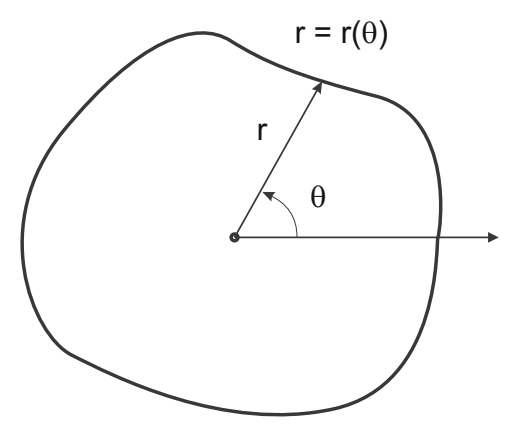

Figure 2. An equilibrium form of biological cluster

Let $C^{m}(2 \pi), m \in \mathbb{N} \cup\{0\}$, denote the Banach space of $2 \pi$-periodic $C^{m}$-smooth functions $r(\theta)$ with the standard norm

$$
\|r\|_{m}=\sum_{k=0}^{m} \max _{\theta \in[0,2 \pi]}\left|r^{(k)}(\theta)\right|,
$$

where $r^{(k)}(\theta)$ denotes the $k$ th derivative of $r(\theta)$ and $r^{(0)}(\theta)=r(\theta)$. It is well known that $C^{m}(2 \pi)$ is continuously embedded into the Hilbert space $L^{2}(2 \pi)$ with the scalar product

$$
\langle f, g\rangle=\int_{0}^{2 \pi} f(\theta) g(\theta) d \theta .
$$

The total energy of the cluster is a sum of three terms:

$$
E=E_{1}+E_{2}+E_{3}
$$

where

$E_{1}$ - the potential energy of stretched elastic boundary of the cluster,

$E_{2}$ - the potential energy of links,

$E_{3}-$ the energy of compressed gas or fluid inside the cluster. 
The first energy $E_{1}$ is proportional to the length of the boundary of the cluster

$$
E_{1}(r, \alpha)=\alpha \int_{0}^{2 \pi} \sqrt{r^{2}(\theta)+r^{\prime 2}(\theta)} d \theta
$$

where $\alpha>0$ is an elasticity coefficient of the boundary. The second energy $E_{2}$ is proportional to the average length of links

$$
E_{2}(r, \beta)=\beta \int_{0}^{2 \pi} r(\theta) d \theta
$$

where $\beta>0$ is an elasticity coefficient of links. The last one is given by

$$
E_{3}(r, \eta, \nu)=\eta S^{-\nu}
$$

where

$$
S=S(r)=\frac{1}{2} \int_{0}^{2 \pi} r^{2}(\theta) d \theta
$$

is the area of the cluster and $\eta>0, \nu>0$ are suitable physical parameters describing the compressed gas or fluid.

Summarizing, the total energy of the cluster is given by

$$
E(r, p)=\int_{0}^{2 \pi}\left(\alpha \sqrt{r^{2}(\theta)+r^{\prime 2}(\theta)}+\beta r(\theta)\right) d \theta+\eta S^{-\nu},
$$

where $p=(\alpha, \beta, \eta, \nu) \in \mathbb{R}_{+}^{4}, r \in C^{m+2}(2 \pi)$ and $r(\theta)>0$ for $\theta \in[0,2 \pi]$.

We see at once that $E$ is smooth. Moreover, an easy computation shows that the Fréchet derivative of $E$ with respect to $r$ is given by

$$
\begin{aligned}
E_{r}^{\prime}(r, p) h= & \int_{0}^{2 \pi} \alpha \frac{r^{3}(\theta)+2 r(\theta) r^{\prime 2}(\theta)-r^{2}(\theta) r^{\prime \prime}(\theta)}{\left(r^{2}(\theta)+r^{\prime 2}(\theta)\right)^{3 / 2}} h(\theta) d \theta \\
& +\int_{0}^{2 \pi}\left(\beta-\frac{\nu \eta}{S^{\nu+1}} r(\theta)\right) h(\theta) d \theta
\end{aligned}
$$

where $p=(\alpha, \beta, \eta, \nu) \in \mathbb{R}_{+}^{4}, r, h \in C^{m+2}(2 \pi)$ and $r(\theta)>0$ for $\theta \in[0,2 \pi]$.

It follows that critical points of the energy functional $E(r, p)$ are $2 \pi$-periodic $C^{m+2}$-smooth positive solutions of the functional-differential equation

$$
\alpha \frac{r^{3}(\theta)+2 r(\theta) r^{\prime 2}(\theta)-r^{2}(\theta) r^{\prime \prime}(\theta)}{\left(r^{2}(\theta)+r^{\prime 2}(\theta)\right)^{3 / 2}}+\beta-\frac{\nu \eta}{S^{\nu+1}} r(\theta)=0 .
$$

We are interested in radially symmetric solutions of (1.3). Substituting $r(\theta) \equiv r$ into (1.3) we get the algebraic equation

$$
\alpha+\beta-\frac{\nu \eta}{\pi^{\nu+1} r^{2 \nu+1}}=0 .
$$

Finally, we get a solution given by

$$
r_{p}=\left(\frac{\nu \eta}{\pi^{\nu+1}(\alpha+\beta)}\right)^{\frac{1}{2 \nu+1}}
$$

for all multiparameters $p=(\alpha, \beta, \eta, \nu) \in \mathbb{R}_{+}^{4}$. Let us remark that a radially symmetric solution $r_{p}$ is decreasing together with the growth of $\alpha$ and $\beta$, i.e. elastic forces attract the boundary of cluster to its center. 

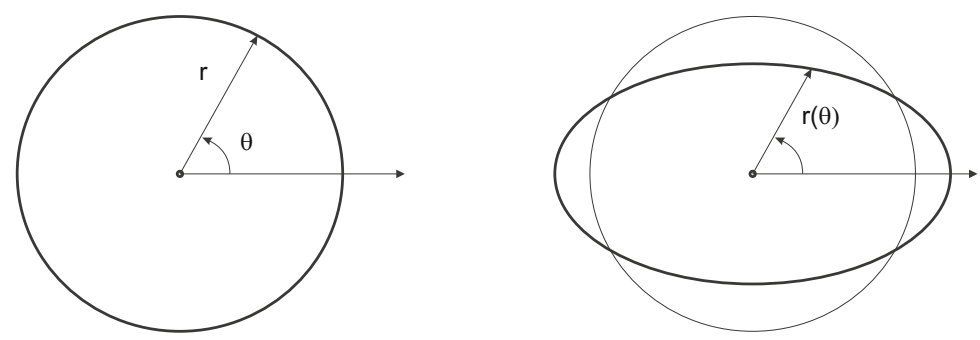

Figure 3. An elliptic transformation

Introduce $\tau=\frac{\beta}{\alpha}, \omega=\frac{\eta}{\alpha}$ and a multiparameter $\mu=(\tau, \omega, \nu)$. It will cause no confusion if we use the same letter $E$ to denote

$$
E(r, \mu)=\int_{0}^{2 \pi}\left(\sqrt{r^{2}(\theta)+r^{\prime 2}(\theta)}+\tau r(\theta)\right) d \theta+\omega S^{-\nu} .
$$

Then (1.3) is equivalent to

$$
\frac{r^{3}(\theta)+2 r(\theta) r^{\prime 2}(\theta)-r^{2}(\theta) r^{\prime \prime}(\theta)}{\left(r^{2}(\theta)+r^{\prime 2}(\theta)\right)^{3 / 2}}+\tau-\frac{\nu \omega}{S^{\nu+1}} r(\theta)=0,
$$

and

$$
r_{\mu}=\left(\frac{\nu \omega}{\pi^{\nu+1}(1+\tau)}\right)^{\frac{1}{2 \nu+1}}
$$

is a radially symmetric solution of the equation (1.5) corresponding to a multiparameter $\mu$. Now our goal is to find critical values of a multiparameter $\mu=(\tau, \omega, \nu)$ such that a solution $r_{\mu}$ loses its symmetry.

Our main result. The breaking of radial symmetry is depending only on the ratio $\tau=\frac{\beta}{\alpha}$ of elasticity coefficients and is not depending on others parameters. If $\tau<3$ then the radially symmetric solution $r(\theta) \equiv r_{\mu}, \mu=(\tau, \omega, \nu)$ given by (1.6) is unique and stable. The elliptic transformation (see Fig. 3) begins when $\tau$ increases from the first critical value $\tau=3$.

More precisely, given any $(\omega, \nu) \in \mathbb{R}_{+}^{2}$ we prove that for each $\tau_{k}=k^{2}-1, k \geq 2$, there exists a branch of non-radially symmetric solutions $(r(t), \tau(t))$ of the equation (1.5), depending on $|t|<\varepsilon$, with

$$
r(t)(\theta)=r_{\left(\tau_{k}, \omega, \nu\right)}+t \cdot \frac{1}{\sqrt{\pi}} \cos (k \theta)+o(|t|)
$$

and $r(0)=r_{\left(\tau_{k}, \omega, \nu\right)}, \tau(0)=\tau_{k}$.

\subsection{The physical origin of the problem}

Our study was motivated by gas balloons. Namely, we are interested in the anatomy and behaviour of the part of a balloon that is actually called an envelope.

The fabric in the envelope is flexible (elastic). It is composed of large vertical sections called gores. Each gore is made up of the same number of horizontal sections called panels. The panels and gores are held together by stitching and by heavy duty 


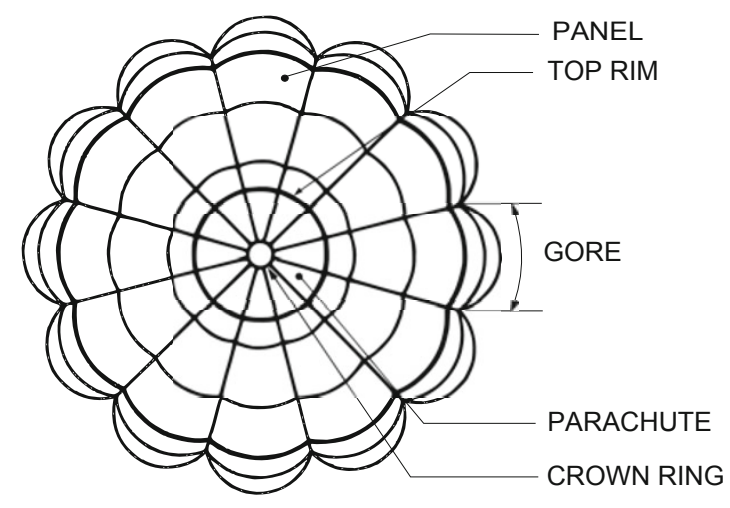

Figure 4. A balloon envelope: view from above

load tapes (webbing similar to the material used in seat belts in automobiles) which help support the weight of the balloon and minimize a strain on the fabric. The top part of the envelope consisting of one panel of each gore is named a parachute (see Fig. 4). The standard parachute possesses a circular deflation port - a crown ring that is closed off by a circular panel which is held sealed during a flight by a flexible hook-and-loop closure.

Gas balloons are inflated with a gas of lower molecular weight than the ambient atmosphere. The most popular gas here is helium.

A biological cluster is a two-dimensional analogue of a gas balloon. This term was proposed by A. Borisovich and H. Treder in [5]. Moreover, we can treat a parachute in a balloon envelope as an example of biological cluster, because its height is much smaller than the length of a top rim - a horizontal tape between the parachute and the rest of envelope. Then a shell of biological cluster is a top rim of parachute, a kernel of biological cluster corresponds to a circular deflation panel, and vertical tapes between panels of parachute are elastic links.

Recently, the interest in symmetry-breaking bifurcation has been increased in connection with works by Avner Friedman and els., where general methods to study free boundary problems were developed (see $[4,8,9,10]$ ). For example, in [9] A. Friedman with F. Reitich and in [10] A. Friedman with B. Hu and J.L. Velázquez considered free boundary problems for a system of two elliptic equations in the plane. They proved the existence of a bifurcation branch of non-radially symmetric solutions with free boundary

$$
R(\varepsilon)(\theta)=R_{0}+\varepsilon \cos (l \theta)+\sum_{n=2}^{\infty} f_{n}(\theta) \varepsilon^{n}
$$

and corresponding parameter

$$
\lambda(\varepsilon)=\lambda_{l}+\sum_{n=1}^{\infty} a_{n} \varepsilon^{n}
$$


where $l \geq 2$ is an integer and $R_{0}>0$ is a free boundary of radially symmetric solution. $\lambda_{l}$ depends on $R_{0}$ and $l$. Their proofs are based on transforming the free boundary into a fixed one, substituting (1.8) and (1.9) into the resulting systems and proceeding to solve inductively with respect to the coefficient of $\varepsilon^{n}, n \in \mathbb{N}$. In [8] M.A. Fontelos and A. Friedman extended the results of [9] and [10] to three dimensions with $\cos (l \theta)$ replaced by the spherical harmonic $Y_{l 0}(\theta)$.

We paid special attention to Friedman's joint work with A. Borisovich [4], where a certain elliptic free boundary problem in the plane was treated. They studied the existence of symmetry-breaking bifurcation from radially symmetric solutions by reducing the problem to one for which classical bifurcation methods may be applied. Their proof is based on the Crandall-Rabinowitz theorem on simple bifurcation points. They suggested that an increasing number of free boundary problems, in particular shell models, might be solved by similar methods.

The investigation of symmetry-breaking bifurcation in a two-dimensional model describing nonlinear deformations of a free elastic shell of biological cluster is of great importance, because it is the introduction to searching three-dimensional models, starting with a special case in which a side surface is formed by the shape of a horizontal crosscut, for example cylindrical balloons.

Let $X$ and $Y$ be Banach spaces. Let $F$ be a continuous map of a neighbourhood of $\left(0, \tau_{0}\right)$ in $X \times \Lambda\left(\Lambda \subset \mathbb{R}^{k}\right.$ an open subset $)$ into $Y$. Suppose that the equation

$$
F(x, \tau)=0
$$

possesses the set of trivial solutions

$$
\Gamma=\left\{(0, \tau): \tau \in \Lambda_{\delta}\left(\tau_{0}\right)\right\}
$$

where $\Lambda_{\delta}\left(\tau_{0}\right)$ denotes a ball of radius $\delta>0$ around $\tau_{0}$ in $\Lambda$.

Let us recall that $\left(0, \tau_{0}\right)$ is called a bifurcation point of $(1.10)$ if there exists another branch of solutions $(x(t), \tau(t))$, depending on $|t|<\varepsilon$, with $\tau(0)=\tau_{0}$ and $x(0)=0$. If the Fréchet derivative of $F$ with respect to $x$ at the point $\left(0, \tau_{0}\right)$ is a Fredholm map of index zero then, by implicit function theorem, the necessary condition for bifurcation at $\left(0, \tau_{0}\right)$ is that dim $\operatorname{ker} F_{x}^{\prime}\left(0, \tau_{0}\right)>0$. General bifurcation theorems provide sufficient conditions for $\left(0, \tau_{0}\right)$ to be a bifurcation point (see for example $[1,6,7,15,17,18,19])$.

A broad class of problems arising in applications is modeled by a nonlinear functional equation as (1.10), where a parameter $\tau$ has a physical interpretation.

The results of this paper were announced without proofs in [5]. We are going to establish symmetry-breaking bifurcation branches of solutions by reducing our free boundary problem to an equation as (1.10) with a nonlinear Fredholm map for which classical and modern bifurcation theory may be applied. We will use the Crandall-Rabinowitz theorem on simple bifurcation points. Moreover, we want to adapt Friedman and collaborator's approach to symmetry breaking bifurcations in free boundary problems (see $[4,8,9,10]$ ). The scheme of application of the CrandallRabinowitz theorem is similar to that in $[2,3,11,12,13,14,16]$. 
The paper is organized as follows. In Section 2 we reformulate the problem of symmetry-breaking bifurcation from radially symmetric solutions of (1.5) to the bifurcation from trivial solutions of an operator equation as (1.10) in appropriate Banach spaces. We prove that an operator $F$ in our model is a nonlinear Fredholm map of index zero and it is a variational gradient of an energy functional. In Section 3 by the use of a variational version of the Crandall-Rabinowitz theorem we prove our main result.

\section{Reformulation of the symmetry-breaking bifurcation problem to one of bifurcation from trivial solutions of a suitable operator equation}

For each $(\omega, \nu) \in \mathbb{R}_{+}^{2}$ the equation (1.5) possesses a family of radially symmetric solutions

$$
\Gamma^{\omega, \nu}=\left\{\left(r_{\mu}, \tau\right): \mu=(\tau, \omega, \nu), \tau \in \mathbb{R}_{+}\right\},
$$

where $r_{\mu}$ is defined by (1.6). Set

$$
\tau_{k}=k^{2}-1, \quad k \geq 2 .
$$

Theorem 2.1. For each $k \geq 2$, there exists a smooth curve of non-radially symmetric solutions $(r(t), \tau(t))$ of (1.5), depending on $|t|<\varepsilon$, with $r(0)=r_{\left(\tau_{k}, \omega, \nu\right)}, \tau(0)=$ $\tau_{k}$ and $r(t)$ of the form (1.7). Thus $\left(r_{\left(\tau_{k}, \omega, \nu\right)}, \tau_{k}\right) \in \Gamma^{\omega, \nu}$ is a symmetry-breaking bifurcation point of the equation (1.5).

It is worth pointing out that problems (as above) coming from elasticity theory are of formidable difficulty and their solving is connected with many technical difficulties.

In this section we are going to reduce the problem of existence of symmetrybreaking bifurcation branches of solutions of (1.5) to the bifurcation from trivial solutions of an equation as (1.10) with a nonlinear Fredholm map of index zero. Moreover, we prove that critical values of bifurcation parameter $\tau$ are $\tau_{k}$.

Let $C_{e}^{m}(2 \pi), m \in \mathbb{N} \cup\{0\}$, be the subspace of $C^{m}(2 \pi)$ of even functions. Set

$$
X=C_{e}^{m+2}(2 \pi) \quad \text { and } \quad Y=C_{e}^{m}(2 \pi) \quad(m \in \mathbb{N} \cup\{0\}) .
$$

Given any $\tau_{0} \in \mathbb{R}_{+}$take $\left(r_{\left(\tau_{0}, \omega, \nu\right)}, \tau_{0}\right) \in \Gamma^{\omega, \nu}$. From now on, $X_{\delta}(0)$ and $\left(\mathbb{R}_{+}\right)_{\delta}\left(\tau_{0}\right)$ denote balls of radius $\delta$ around 0 in $X$ and $\tau_{0}$ in $\mathbb{R}_{+}$, respectively. For $\varrho \in X_{\delta}(0)$ and $\tau \in\left(\mathbb{R}_{+}\right)_{\delta}\left(\tau_{0}\right)$ define

$$
r(\theta)=r_{\mu}+\varrho(\theta)
$$

where $\mu=(\tau, \omega, \nu)$ is considered as a function of $\tau$. ( $\delta$ is sufficiently small such that $r(\theta)>0$.) Remark that $r(\theta)$ is a small perturbation in $X$ from $r_{\mu}$ given by (1.6).

Substituting (2.3) in (1.4), we get the energy functional $\hat{E}$ given by

$$
\hat{E}(\varrho, \mu)=\int_{0}^{2 \pi}\left(\sqrt{\left(r_{\mu}+\varrho\right)^{2}+\varrho^{\prime 2}}+\tau\left(r_{\mu}+\varrho\right)\right) d \theta+\omega \hat{S}^{-\nu},
$$


where $\varrho \in X_{\delta}(0), \tau \in\left(\mathbb{R}_{+}\right)_{\delta}\left(\tau_{0}\right)$ and $\mu=(\tau, \omega, \nu)$, and

$$
\hat{S}=\hat{S}(\varrho, \mu)=\frac{1}{2} \int_{0}^{2 \pi}\left(r_{\mu}+\varrho\right)^{2} d \theta \text {. }
$$

Moreover, the Fréchet derivative of $\hat{E}$ with respect to $\varrho$ is given by

$$
\begin{aligned}
\hat{E}_{\varrho}^{\prime}(\varrho, \mu) h= & \int_{0}^{2 \pi} \frac{\left(r_{\mu}+\varrho\right)^{3}+2\left(r_{\mu}+\varrho\right) \varrho^{2}-\left(r_{\mu}+\varrho\right)^{2} \varrho^{\prime \prime}}{\left\{\left(r_{\mu}+\varrho\right)^{2}+\varrho^{\prime 2}\right\}^{3 / 2}} h d \theta \\
& +\int_{0}^{2 \pi}\left(\tau-\frac{\nu \omega}{\hat{S}^{\nu+1}}\left(r_{\mu}+\varrho\right)\right) h d \theta .
\end{aligned}
$$

Let $\hat{F}: X_{\delta}(0) \times\left(\mathbb{R}_{+}\right)_{\delta}\left(\tau_{0}\right) \rightarrow Y$ be given by

$$
\hat{F}(\varrho, \mu)=\frac{\left(r_{\mu}+\varrho\right)^{3}+2\left(r_{\mu}+\varrho\right) \varrho^{\prime 2}-\left(r_{\mu}+\varrho\right)^{2} \varrho^{\prime \prime}}{\left\{\left(r_{\mu}+\varrho\right)^{2}+\varrho^{\prime 2}\right\}^{3 / 2}}+\tau-\frac{\nu \omega}{\hat{S}^{\nu+1}}\left(r_{\mu}+\varrho\right) .
$$

Of course, $\hat{F}$ is smooth. By the above, critical points of $\hat{E}(\varrho, \mu)$ are $2 \pi$-periodic $C^{m+2}$-smooth even solutions of the equation

$$
\hat{F}(\varrho, \mu)=0 .
$$

According to (2.5), we have

Lemma 2.2. For each $\tau \in\left(\mathbb{R}_{+}\right)_{\delta}\left(\tau_{0}\right), \hat{F}(., \mu): X_{\delta}(0) \rightarrow Y$ is a variational gradient of $\hat{E}(., \mu): X_{\delta}(0) \rightarrow \mathbb{R}$ with respect to the scalar product in $L^{2}(2 \pi)$, i.e.

$$
\hat{E}_{\varrho}^{\prime}(\varrho, \mu) h=\langle\hat{F}(\varrho, \mu), h\rangle
$$

for all $h \in X$.

The equation (2.7) possesses a trivial family of solutions

$$
\hat{\Gamma}^{\omega, \nu}=\left\{(0, \tau) \in X \times \mathbb{R}_{+}: \tau \in\left(\mathbb{R}_{+}\right)_{\delta}\left(\tau_{0}\right)\right\} .
$$

In order to establish the existence of a symmetry-breaking bifurcation branch of solutions of $(1.5)$ at $\left(r_{\left(\tau_{0}, \omega, \nu\right)}, \tau_{0}\right)$ we will study the bifurcation from trivial solutions of $(2.7)$ at $\left(0, \tau_{0}\right)$.

The task is now to prove the lemma below.

Lemma 2.3. For each $\tau \in\left(\mathbb{R}_{+}\right)_{\delta}\left(\tau_{0}\right), \hat{F}_{\varrho}^{\prime}(0, \mu): X \rightarrow Y$ is a Fredholm map of index 0 .

Proof. Fix $\tau \in\left(\mathbb{R}_{+}\right)_{\delta}\left(\tau_{0}\right)$. An easy computation shows that

$$
\hat{F}_{\varrho}^{\prime}(0, \mu) h=-\frac{1}{r_{\mu}} h^{\prime \prime}-\frac{\nu \omega}{\pi^{\nu+1} r_{\mu}{ }^{2 \nu+2}}\left(h-\frac{\nu+1}{\pi} \int_{0}^{2 \pi} h(\theta) d \theta\right)
$$

for each $h \in X$. The map $\hat{F}_{\varrho}^{\prime}(0, \mu): X \rightarrow Y$ may be written as a sum

$$
\hat{F}_{\varrho}^{\prime}(0, \mu)=A+B
$$

where

$$
A(h)=-\frac{1}{r_{\mu}} h^{\prime \prime}
$$


and

$$
B(h)=-\frac{\nu \omega}{\pi^{\nu+1} r_{\mu}{ }^{2 \nu+2}}\left(h-\frac{\nu+1}{\pi} \int_{0}^{2 \pi} h(\theta) d \theta\right) .
$$

To finish the proof it suffices to show that $A: X \rightarrow Y$ is a Fredholm map of index 0 , and $B: X \rightarrow Y$ is completely continuous. It is clear that $A$ is a linear continuous mapping with $\operatorname{ker} A=\{h(\theta)=C: C \in \mathbb{R}\}$, and so $\operatorname{dim} \operatorname{ker} A=1$. We have

$$
X=\operatorname{ker} A \oplus\left\{h \in X: \int_{0}^{2 \pi} h(\theta) d \theta=0\right\},
$$

and

$$
Y=\{h(\theta)=C: C \in \mathbb{R}\} \oplus \operatorname{im} A .
$$

From this we see that $\operatorname{codimim} A=1$, and consequently $A$ is a Fredholm map of index 0 . Now let us consider the operator $B$ as a composition $B=B_{1} \circ B_{2}$, where $B_{2}: X \rightarrow X$ is defined by

$$
B_{2}(h)=-\frac{\nu \omega}{\pi^{\nu+1} r_{\mu}{ }^{2 \nu+2}}\left(h-\frac{\nu+1}{\pi} \int_{0}^{2 \pi} h(\theta) d \theta\right)
$$

and $B_{1}: X \rightarrow Y$ is the natural embedding of $X$ into $Y$. As $B_{2}$ is a continuous mapping and $B_{1}$ is a completely continuous one, $B$ is also a completely continuous map.

By the implicit function theorem the necessary condition for bifurcation from trivial solutions of $(2.7)$ at $\left(0, \tau_{0}\right)$ is that $\operatorname{dim} \operatorname{ker} \hat{F}_{\varrho}^{\prime}\left(0, \tau_{0}, \omega, \nu\right)>0$.

To find critical values of bifurcation parameter we have to solve the equation

$$
\hat{F}_{\varrho}^{\prime}(0, \tau, \omega, \nu) h=0
$$

with two additional conditions

$$
\int_{0}^{2 \pi} h(\theta) \cos \theta d \theta=0
$$

and

$$
\int_{0}^{2 \pi} h(\theta) d \theta=0
$$

The assumption (2.13) implies a loss of radial symmetry. The evenest of $h(\theta)$ and (2.12) exclude a displacement of mass center of the cluster. The conditions (2.12) and (2.13) exclude $h(\theta)=\cos (\theta)$ and $h(\theta)=$ const $\neq 0$.

Moreover, the assumption (2.13) simplifies the equation (2.11) to

$$
-\frac{1}{r_{\mu}} h^{\prime \prime}-\frac{\nu \omega}{\pi^{\nu+1} r_{\mu}^{2 \nu+2}} h=0 .
$$

We take the bifurcation mode $e_{k}(\theta)=\frac{1}{\sqrt{\pi}} \cos (k \theta)$ for $k \geq 2$. We obtain

$$
\frac{1}{\sqrt{\pi}} \cdot \frac{1}{r_{\mu}} \cos (k \theta)\left(k^{2}-\frac{\nu \omega}{\pi^{\nu+1} r_{\mu}^{2 \nu+1}}\right)=0 .
$$

By (1.6),

$$
r_{\mu}^{2 \nu+1}=\frac{\nu \omega}{\pi^{\nu+1}(1+\tau)},
$$


and, in consequence,

$$
k^{2}-1-\tau=0
$$

which implies

$$
\tau=\tau_{k}=k^{2}-1, \quad k \geq 2 .
$$

Theorem 2.4. Substituting $\tau_{k}(k \geq 2)$ into $\tau_{0}$. Then $\left(0, \tau_{k}\right)$ is a bifurcation point of (2.7) and the solution set of (2.7) in a neighbourhood of this point is the sum of $\hat{\Gamma}^{\omega, \nu}$ and a smooth curve $(\varrho(t), \tau(t))$, parametrized by $|t|<\varepsilon$, such that $\varrho(0)=0, \tau(0)=$ $\tau_{k}$ and

$$
\varrho(t)(\theta)=t \cdot \frac{1}{\sqrt{\pi}} \cos (k \theta)+o(|t|) .
$$

\section{Proof of Theorem 2.4}

In this section we prove Theorem 2.4. Our proof is based on the Crandall-Rabinowitz theorem on simple bifurcation points (see [7]). More precisely, we will apply a gradient (variational) version of the Crandall-Rabinowitz theorem due to A.Yu. Borisovich (see $[2,3])$. For the convenience of the reader we state this theorem. For the proof we refer to [13].

Theorem 3.1. Assume that $H$ is a Hilbert space with a scalar product $(., .)_{H}$. Let $X$ and $Y$ be Banach spaces continuously embedded in $H$. Suppose that a $C^{r}$-operator $F: X_{\delta}(0) \times \mathbb{R}_{\delta}\left(\tau_{0}\right) \rightarrow Y$ and a $C^{r+1}$-functional $E: X_{\delta}(0) \times \mathbb{R}_{\delta}\left(\tau_{0}\right) \rightarrow \mathbb{R}$, where $r \geq 2$, satisfy the following conditions:

1. $F(0, \tau)=0$ for $\tau \in \mathbb{R}_{\delta}\left(\tau_{0}\right)$,

2. $\operatorname{dim} \operatorname{ker} F_{x}^{\prime}\left(0, \tau_{0}\right)=1, F_{x}^{\prime}\left(0, \tau_{0}\right) e=0,(e, e)_{H}=1$,

3. $\operatorname{codimim} F_{x}^{\prime}\left(0, \tau_{0}\right)=1$,

4. $E_{x}^{\prime}(x, \tau) h=(F(x, \tau), h)_{H}$ for $(x, \tau) \in X_{\delta}(0) \times \mathbb{R}_{\delta}\left(\tau_{0}\right)$ and $h \in X$,

5. $E_{x x \tau}^{\prime \prime \prime}\left(0, \tau_{0}\right) e e \neq 0$.

Then $\left(0, \tau_{0}\right)$ is a bifurcation point of the equation

$$
F(x, \tau)=0 .
$$

In fact, the solution set of this equation in a certain neighbourhood of $\left(0, \tau_{0}\right)$ consists of the curve $\Gamma_{1}=\left\{(0, \tau): \tau \in \mathbb{R}_{\delta}\left(\tau_{0}\right)\right\}$ and a $C^{r-2}$-curve $\Gamma_{2}$, intersecting only at $\left(0, \tau_{0}\right)$. Moreover, if $r \geq 3$, the curve $\Gamma_{2}$ can be parametrized by a variable $t,|t| \leq \varepsilon$, as

$$
\Gamma_{2}=\left\{(x(t), \tau(t)): t \in \mathbb{R}_{\varepsilon}(0)\right\},
$$

where $x(0)=0, \tau(0)=\tau_{0}$ and $x^{\prime}(0)=e$.

Proof of Theorem 2.4. To finish the proof it is sufficient to show that

$$
\hat{E}_{\varrho \varrho \tau}^{\prime \prime \prime}\left(0, \tau_{k}, \omega, \nu\right) e_{k} e_{k} \neq 0 .
$$

From Lemma 2.2 it follows that

$$
\hat{E}_{\varrho \varrho}^{\prime \prime}(0, \mu) h g=\left\langle\hat{F}_{\varrho}^{\prime}(0, \mu) h, g\right\rangle .
$$


Applying (2.9) we have

$$
\begin{aligned}
\hat{E}_{\varrho \varrho}^{\prime \prime}(0, \mu) h g & =\int_{0}^{2 \pi}\left(-\frac{1}{r_{\mu}} h^{\prime \prime}(\theta)-\frac{\nu \omega}{\pi^{\nu+1} r_{\mu}{ }^{2 \nu+2}} h(\theta)\right) g(\theta) d \theta+ \\
& +\int_{0}^{2 \pi}\left(\frac{\nu(\nu+1) \omega}{\pi^{\nu+2} r_{\mu}^{2 \nu+2}} \int_{0}^{2 \pi} h(\bar{\theta}) d \bar{\theta}\right) g(\theta) d \theta .
\end{aligned}
$$

Substituting $h=g=e_{k}$ to (3.1), we obtain

$$
\hat{E}_{\varrho \varrho}^{\prime \prime}(0, \mu) e_{k} e_{k}=\frac{1}{r_{\mu}}\left(k^{2}-1-\tau\right) .
$$

Differentiating (3.2) with respect to $\tau$ we get

$$
\hat{E}_{\varrho \varrho \tau}^{\prime \prime \prime}(0, \mu) e_{k} e_{k}=\left(\frac{\pi^{\nu+1}(1+\tau)}{\nu \omega}\right)^{\frac{1}{2 \nu+1}}\left(\frac{k^{2}}{(2 \nu+1)(1+\tau)}-\frac{1}{2 \nu+1}-1\right) .
$$

Finally, for critical values of parameter $\tau$ we have

$$
\hat{E}_{\varrho \varrho \tau}^{\prime \prime \prime}\left(0, \tau_{k}, \omega, \nu\right) e_{k} e_{k}=-\sqrt[2 \nu+1]{\frac{\pi^{\nu+1} k^{2}}{\nu \omega}}<0, \quad k \geq 2,
$$

which completes the proof.

\section{References}

[1] S. S. Antman, Nonlinear Problems of Elasticity. Appl. Math. Sci. 107, Springer-Verlag, New York, 1995.

[2] A. Yu. Borisovich, J. Dymkowska, Cz. Szymczak, Buckling and postcritical behaviour of the elastic infinite plate strip resting on linear elastic foundation. J. Math. Anal. Appl. 307 (2005), no. 2, $480-495$.

[3] A. Yu. Borisovich, J. Janczewska, Stable and unstable bifurcation in the von Kármán problem for a circular plate. Abstr. Appl. Anal. 2005, no. 8, 889 - 899.

[4] A. Yu. Borisovich, A. Friedman, Symmetry-breaking bifurcations for free boundary problems. Indiana Univ. Math. J. 54 (2005), no. 3, 927 - 947.

[5] A. Yu. Borisovich, H. Treder (after marriage H. Guze), Symmetry-breaking bifurcation for free elastic shell of biological cluster. AIP Conference Proceedings 936 (2007), 90-92.

[6] S. N. Chow, J. K. Hale, Methods of Bifurcation Theory. Springer-Verlag, New YorkBerlin, 1982.

[7] M. G. Crandall, P. H. Rabinowitz, Bifurcation from simple eigenvalues. J. Funct. Anal. 8 (1971), $321-340$.

[8] M. A. Fontelos, A. Friedman, Symmetry-breaking bifurcations of free boundary problems in free dimensions. Asymptot. Anal. 35 (2003), no. 3-4, $187-206$.

[9] A. Friedman, F. Reitich, Symmetry-breaking bifurcation of analytic solutions to free boundary problems: an application to a model of tumor growth. Trans. Amer. Math. Soc. 353 (2001), no. 4, $1587-1634$.

[10] A. Friedman, B. Hu, J. L. Velázquez, A Stefan problem for a protocell model with symmetry-breaking bifurcations of analytic solutions. Interfaces Free Bound. 3 (2001), no. $2,143-199$. 
[11] J. Janczewska, Bifurcation in the solution set of the von Kármán equations of an elastic disk lying on an elastic foundation. Ann. Polon. Math. 77 (2001), no. 1, 53-68.

[12] J. Janczewska, The necessary and sufficient condition for bifurcation in the von Kármán equations. NoDEA Nonlinear Differential Equations Appl. 10 (2003), no. 1, 73 - 94.

[13] J. Janczewska, Local properties of the solution set of the operator equation in Banach spaces in a neighbourhood of a bifurcation point. Cent. Eur. J. Math. 2 (2004), no. 4, $561-572$.

[14] J. Janczewska, Multiple bifurcation in the solution set of the von Kármán equations with $S^{1}$-symmetries. Bull. Belg. Math. Soc. Simon Stevin 15 (2008), no. 1, $109-126$.

[15] N. F. Morozov, Selected Two-Dimensional Problems of Elasticity Theory. Univ. Press, Leningrad, 1978 (in Russian).

[16] Yu. Morozov, The study of the nonlinear model which desribes the equilibrum forms, fundamental frequencies and modes of oscillations of a finite beam on an elastic foundation. Ph. D. Thesis, Appl. Math. Department, Voronezh University, 1998 (in Russian).

[17] L. Nirenberg, Topics in Nonlinear Functional Analysis. Courant Inst. Math. Sci., 1974.

[18] Yu. I. Sapronov, Branching of Solutions of Smooth Fredholm Equations, Equations on Manifolds. 60 - 82, Novoe Global. Anal.,Voronezh. Gos. Univ., Voronezh, 1982 (in Russian).

[19] V. A. Trenogin, M. M. Vainberg, Theory of Branching of Solutions of Non-linear Equations. Noordhoff International Publishing, Leyden, 1974.

Hanna Guze

Mathematics Teaching and Distance Learning Center

Gdańsk University of Technology

Narutowicza 11/12

80-233 Gdańsk

Poland

e-mail: hanna.guze@pg.gda.pl

Joanna Janczewska

Faculty of Applied Physics and Mathematics

Gdańsk University of Technology

Narutowicza 11/12

80-233 Gdańsk

Poland

e-mail: janczewska@mif.pg.gda.pl

Received: January 26, 2014.

Open Access This article is distributed under the terms of the Creative Commons Attribution License which permits any use, distribution, and reproduction in any medium, provided the original author(s) and the source are credited. 